\title{
Scalarizing functions in solving multi-objective problem-an evolutionary approach
}

\author{
D. Vasumathi, S. Thangavelu
}

Department of Computer science and Engineering, Amrita school of engineering, Amrita Vishwa Vidyapeetham, India

\begin{tabular}{l}
\hline \hline Article Info \\
\hline Article history: \\
Received Jul 6, 2018 \\
Revised Dec 5, 2018 \\
Accepted Dec 22, 2018 \\
\hline Keywords: \\
Differential evolution \\
Indicator functions \\
MOEA framework \\
Multi-objective problems \\
Scalarizing functions \\
\hline
\end{tabular}

\begin{abstract}
Scalarizing functions had long been observed for optimization of multiobjective problems. Scalarizing functions on multi-objective problem along with Differential Evolution (DE) algorithm variants had been used to analyze the effect of scalarizing functions. The main purpose is to find the better scalarizing function which can be applied for optimization. The effective solution of the multi-objective problem depends on the various factors like the $\mathrm{DE}$ algorithm and the scalarizing functions used. Multi objective evolutionary algorithm (MOEA) framework in java had been used for performing the analysis. The Obtained results showed that Tchebysheff scalarization function performs better than the other scalarizing functions on various indicator functions used.
\end{abstract}

Copyright $(2) 2019$ Institute of Advanced Engineering and Science. All rights reserved.

\section{Corresponding Author:}

S. Thangavelu,

Department of Computer science and Engineering,

Amrita Vishwa Vidyapeetham, India.

Email: s_thangavel@cb.amrita.edu

\section{INTRODUCTION}

Recently, Differential evolution has attracted considerable research interest in multi-objective optimization in the domain of evolutionary algorithm. On comparison with traditional algorithms, DE is found to be performing better on different problems. The main research issue focuses on performance evaluation, fitness assignment and diversity maintenance where our main goal is performance evaluation. Recently some works on analyzing performance are also done [1].

A Multi-objective problem (MOP) is defined as an optimization problem with several objective functions with decision variables in the feasible region $\Omega$. Mathematically it is given in equation (1)

$$
\min F(x)=\left(f_{1}(x), f_{2}(x) \ldots f_{m}(x)\right)
$$

where $x \in \Omega$

A problem which has one or more objective is called multi-objective problem. Scalarizing functions, in general, are used for decomposing a multi-objective problem into several single objective problems. Objective functions are denoted by $f(x)$. Each multi-objective problem will have several objective functions and several decision variables.

Differential Evolution (DE) is an evolutionary algorithm for optimizing a problem iteratively for improving the quality of the candidate solution. Differential evolution has been found to perform better on stability [2] thus, differential evolution is used for performing experiments for solving MOP using scalarizing functions. A number of scalarizing functions exist in literature which are used for performing experiments on different multi-objective optimization algorithms. Penalty bound intersection (PBI), Tchebysheff and weighted sum are commonly used scalarizing functions for any experiments. Hence, these are used on the experiments performed in this analysis. 
DE steps includes initialization, mutation, recombination and selection. Initialization part assigns lower and upper bound to each of the target variable $x$ in candidates of the population and generate the candidates within that boundary. Mutation generates donor vector $v$ based on weighted difference of two different candidates and adding with another candidate, in which the method of selection of candidate decides the type of DE variant as DE/rand or DE/best. A Trial vector is produced, which is denoted by $u$ produced from donor vector and target vector. Selection mechanism selects better solution either from trial vector or target vector.

After DE is applied and final population is found, they are applied with indicator functions for analyzing the performance of scalarizing functions. ZDT1 benchmark problem has been used in this analysis with four different DE algorithmic variants such as $D E / \mathrm{rand} / 1 / \mathrm{bin}, D E / \mathrm{rand} / 2 / \mathrm{bin}$, DE/best/1/bin and DE/best/2/bin with the scalarizing functions like PBI, Weighted sum, Tchebysheff, Modified Tchebysheff [3]. The indicator functions such as hyper volume and Inverted generational distance (IGD) are used for analyzing the performance of scalarizing functions on solving MOPs and Tchebysheff scalarization function is found to perform better than other scalarizing functions.

The division of the remaining paper is as follows section 2 includes literature survey which includes basic concepts of multi-objective problem, scalarizing functions such as Tchebysheff (TS), Weighted Sum (WS), Penalty Bound Intersection (PBI), Modified Tchebysheff (MTS), variants of DE algorithms and indicator for performance evaluation. Section 3 includes experimental procedure, Section 4 includes experimental results and discussion and section 5 contain the conclusion.

\section{LITERATURE SURVEY}

\subsection{DE variants}

a) DE/rand/p

It is a general scheme where solutions are picked randomly. For each solution $x^{(k)}$, where $k$ varies from 1 to $\mathrm{N}$ vector $\mathrm{y}$ is obtained by equation (3)

$$
y=x^{\left(r_{1}\right)}+F\left(\sum_{i=1}^{p}\left(x^{\left(r_{2, t}\right)}-x^{\left(r_{3, t}\right)}\right)\right)
$$

In the above equation, $r_{1}, r_{2, t}, r_{3, t}$ lies in 1 to $\mathrm{N}$ and they are unique and mutually exclusive. $\mathrm{F}$ is a constant factor within $[0,2]$. Number of weighted difference is given by $p$. When $p$ is 1 , it is DE/rand/ 1 where $r_{1}$ is randomly chosen. $y$ is the donor vector obtained in mutation.

\section{b) DE/best/p}

This DE scheme works similarly to rand/p scheme except that value of $r_{1}$ is the minimum value of the variables in solution for minimization problems. If value of $p$ is changed to 2 then it is DE/best $/ 2$. For each solution $x^{(k)}$, where $\mathrm{k}$ varies from 1 to $\mathrm{N}$ vector $\mathrm{y}$ is obtained by the equation (4)

$$
y=x^{(\text {best })}+F\left(\sum_{i=1}^{p}\left(x^{\left(r_{2, t}\right)}-x^{\left(r_{3, t}\right)}\right)\right)
$$

In the above equation, $r_{1}, r_{2, t}, r_{3, t}$ lies in 1 to $\mathrm{N}$ and they are unique and mutually exclusive. Constant factor $\mathrm{F}$, is within the range of 0 to 2. $p$ is number of weighted difference.

\subsection{Scalarizing function}

Scalarizing functions perform operations on individual objectives with each candidate of a population and produces a single fitness value. Fitness is compared among the parent and children and better solution goes to the next generation and refining happens for each generation producing a better Pareto front.

\section{a) Weighted sum}

This is the basic scalarizing function used on almost every scalarizing experiments on different papers [1], [7] and [8]. Weighted sum multiplies weight along with objective. Each weight should be chosen in a way that sum of weights should be equal to 1 and any weight should lie between 0 and 1 . It is generally represented by the equation (5)

$$
\operatorname{Minimize} F(X)=\sum_{i=1}^{M} w_{i} f_{i}(x)
$$

Where $w$ is weight and $f(x)$ is objective function. 


\section{b) Tchebysheff}

It is slightly different from weighted sum method in which fitness is calculated with each objective getting subtracted from the minimum value of the objective in the whole population which will be multiplied along with weight where any random weight will be within range 0 to 1 [3]. Tchebysheff decomposition is mathematically given by the equation (6)

$$
\text { minimize } \max _{i=1, \ldots k}\left[w_{i}\left(f_{i}(x)-z_{i}^{*}\right)\right]
$$

where $w_{i}$ represent weight, $f_{i}(x)$ is objective function and $z_{i}^{*}$ is minimum value of the objective function.

\section{c) Penalty Bound Intersection (PBI)}

This method calculates fitness based on sum of $d 1$ and $d 2$ which corresponds to projection vector length $\left(f(x)-z^{*}\right)$ on weight vector $w$ and perpendicular distance from $f(x)$ to $w$. $\theta$ is penalty factor which is multiplied with $d_{2}$ and added along with d1. Equation (7) denotes the measurement of fitness using PBI.

$$
\begin{aligned}
& \min g^{p b i}(x \mid w, z *)=d_{1}+\theta d_{2} \\
& \text { s. t } x \in \Omega_{x} \\
& d_{1}=\frac{\left\|\left(f(x)-z^{*}\right)^{T} w\right\|}{\|w\|} \\
& d_{2}=\left\|f(x)-\left(z^{*}+d_{1} \frac{w}{\|w\|}\right)\right\|
\end{aligned}
$$

$z^{*}$ represents minimum value of the objective function. $d_{1}$ measures convergence and $d_{2}$ represents diversity. From [7] we come to know that PBI is able to converge convex Pareto front large diversity corresponds to large $\theta$ values and less value corresponds to convergence.

\section{d) Modified Tchebysheff:}

According to [3] modified Tchebysheff is same as weighted Tchebysheff except that weights are divided instead of being multiplied. It is given mathematically in equation (8)

$$
\min _{x \in \Omega} g^{m t c h}\left(F(x) \mid w, z^{*}\right)=\max _{1 \leq i \leq m}\left\{\frac{f_{i}(x)-z_{i}^{*}}{w_{i}}\right\}
$$

it is easy to handle nonlinear relationships by making this modification to conventional Tchebysheff [3].

\section{RESEARCH METHOD}

In this paper, four scalarizing functions namely PBI, Tchebysheff, Modified Tchebysheff and Weighted sum are used for experiments with different DE variants. All the experiments are performed on ZDT1, a convex Pareto front problem with uniform weight $w 0.5$ and 0.5 . Crossover rate, CR and scaling factor, $\mathrm{F}$ of the crossover and mutation operations of DE algorithm have been set with 0.1 and 0.5 respectively. Population size is set as 100 and each candidate is defined with 30 decision variables. The stopping criteria of the algorithm has been set as 10000 function evaluations. The total number of runs used is 5 for all variants of different scalarizing functions, As shown in Figure 1.

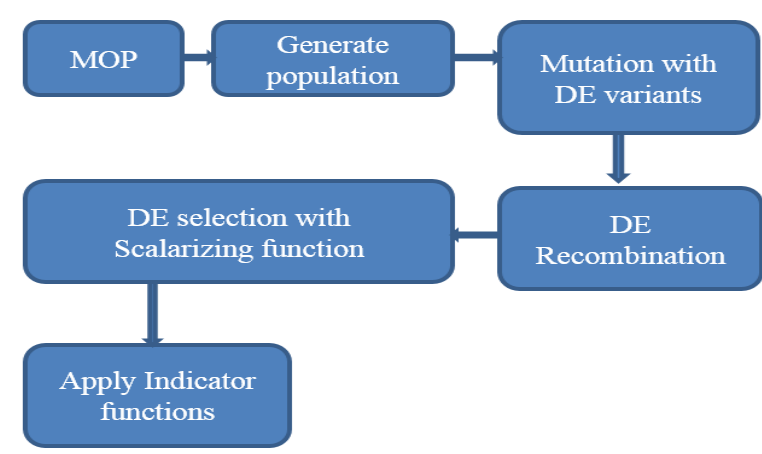

Figure 1. Procedure for performing the experiment 
The following Figure 1 represents the procedure used for performing the analysis. To compare the performance inverted generational distance and hyper volume has been used in order to measure the quality of non-dominated solution obtained in different variants because of different scalarization function.

\section{RESULTS AND DISCUSSION}

The performance results of the various DE algorithms on the metrics IGD and HV are grouped for each scalarizing functions.

\subsection{Analysis with variation of indicator functions}

a) IGD for weighted sum

Figure 2 shows the results of different DE variants viz. DE/rand/1, DE/rand/2, DE/best/1 and $\mathrm{DE} / \mathrm{best} / 2$, on IGD metrics using the scalarizing function weighted sum.

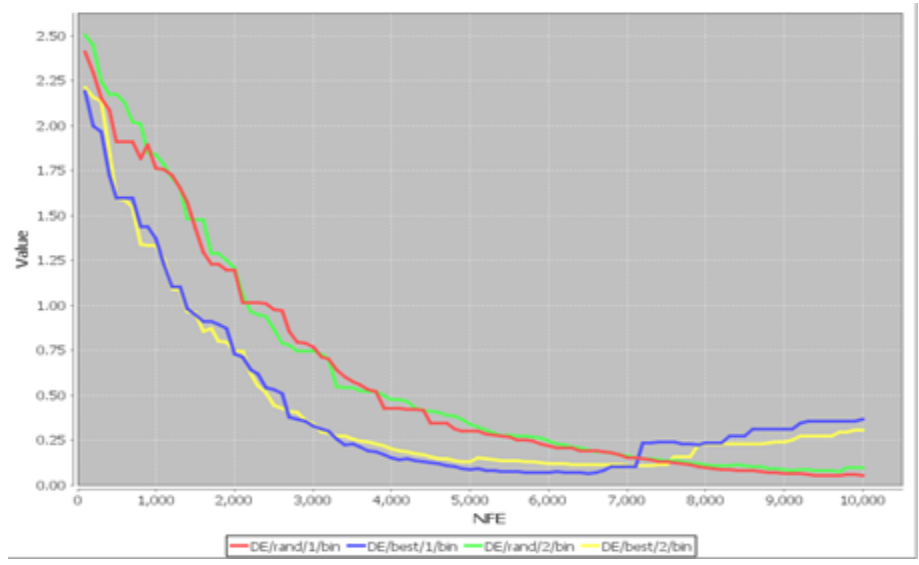

Figure 2. Comparison of DE variants with IGD metrics and weighted sum scalarization function

DE/best/1 starts performing better on initial part of function evaluation but DE/best/2/bin starts performing better from function evaluation 500 . DE/rand/2 and DE/rand/1 initially does not perform well but at the end of mid of 7800 evaluations DE/rand/1 and rand/2 starts performing well and at the end of 10000 evaluations DE/rand/1 converging towards the solution than other DE variants.

\section{b) IGD Modified Tchebysheff}

Figure 3 shows the results of different DE variants viz. DE/rand/1, DE/rand/2, DE/best/1 and DE/best/2, on IGD metrics using the scalarizing function Modified Tchebysheff

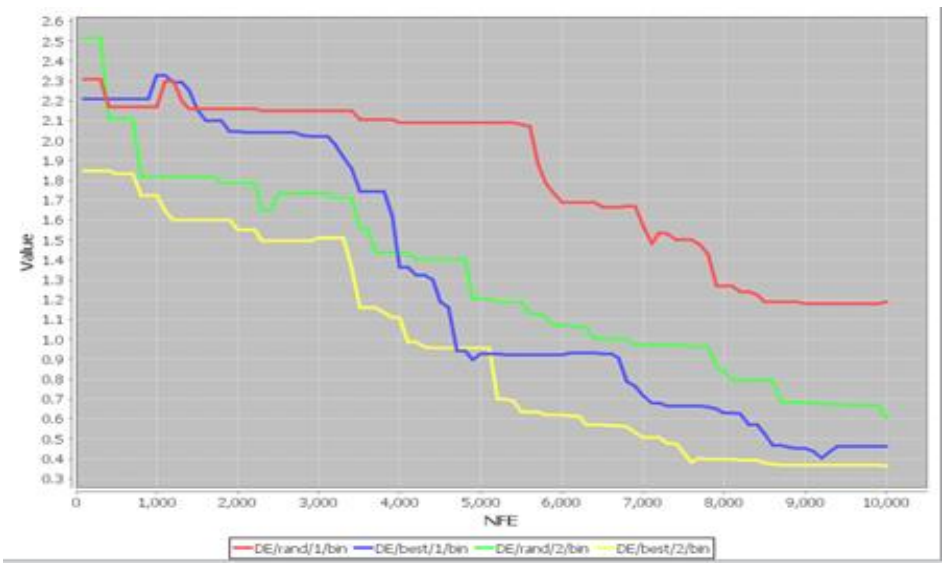

Figure 3. Comparison of DE variants with IGD metrics and modified Tchebysheff scalarization function 
Applying IGD on modified tchebysheff DE/best/2 starts to perform better than all other variants. In 500 function evaluation DE/rand/2 starts to perform better than best/1 and rand/1. At 4000 evaluations best/1 and rand/2 clashes and best/1 starts performing better there after. DE/rand/1 performs better till 1000 function evaluation and after that $\mathrm{DE} / \mathrm{best} / 1$ and rand/2 starts performing better. At the end overall best/2 performs better.

\section{c) IGD Penalty Bound Intersection}

Figure 4 shows the results of different DE variants viz. DE/rand/1, DE/rand/2, DE/best/1 and DE/best/2, on IGD metrics using the scalarizing function Modified Tchebysheff.

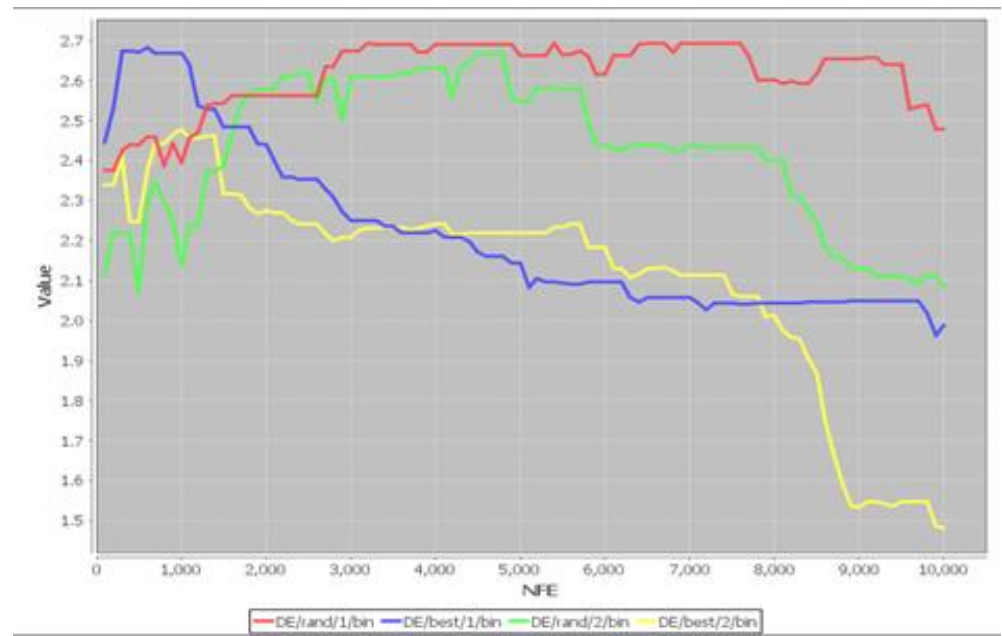

Figure 4. Comparison of DE variants with IGD metrics and PBI

According to PBI, DE/rand/2 starts performing better initially but DE/best/2 overtakes it at 1500 function evaluation. DE/rand/1 tries to perform better than best $/ 2$ at around end of 700 evaluations. DE/best $/ 2$ starts performing better than best/ 1 at 7800 evaluations and at the end DE/best/2 performs better according to IGD on penalty factor of theta 1.0

\section{d) IGD Tchebysheff}

Figure 5 shows the results of different DE variants viz. DE/rand/1, DE/rand/2, DE/best/1 and DE/best/2, on IGD metrics using the scalarizing function Tchebysheff.

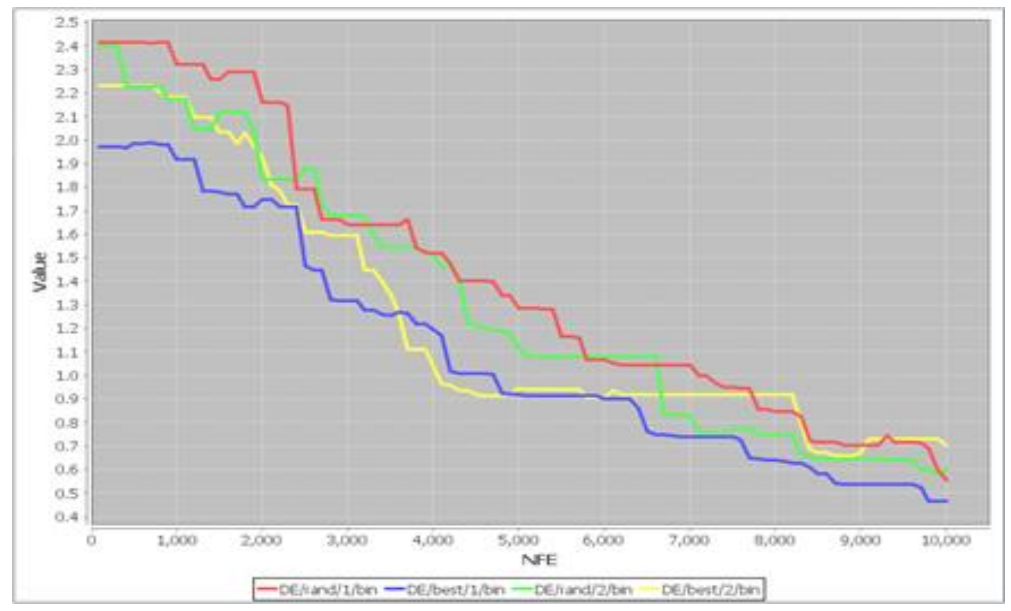

Figure 5. Comparison of DE variants with IGD metrics and Tchebysheff 
Applying IGD metrics on Tchebysheff scalarization function, DE/best/1 starts to perform better from the first and at the mid of 2300 function evaluation DE/best/1 and DE/best/2 overlaps and in the middle of 3500 function evaluation DE/best/2 starts to perform better and at the end DE/best/1 performs better than all other variants.

\subsection{Analysis with Variation in scalarizing functions}

\subsubsection{Weighted sum}

Figure 6 shows the results of different DE variants viz. DE/rand/1, DE/rand/2, DE/best/1 and $\mathrm{DE} / \mathrm{best} / 2$, on $\mathrm{HV}$ and IGD metrics using the scalarizing function weighted sum

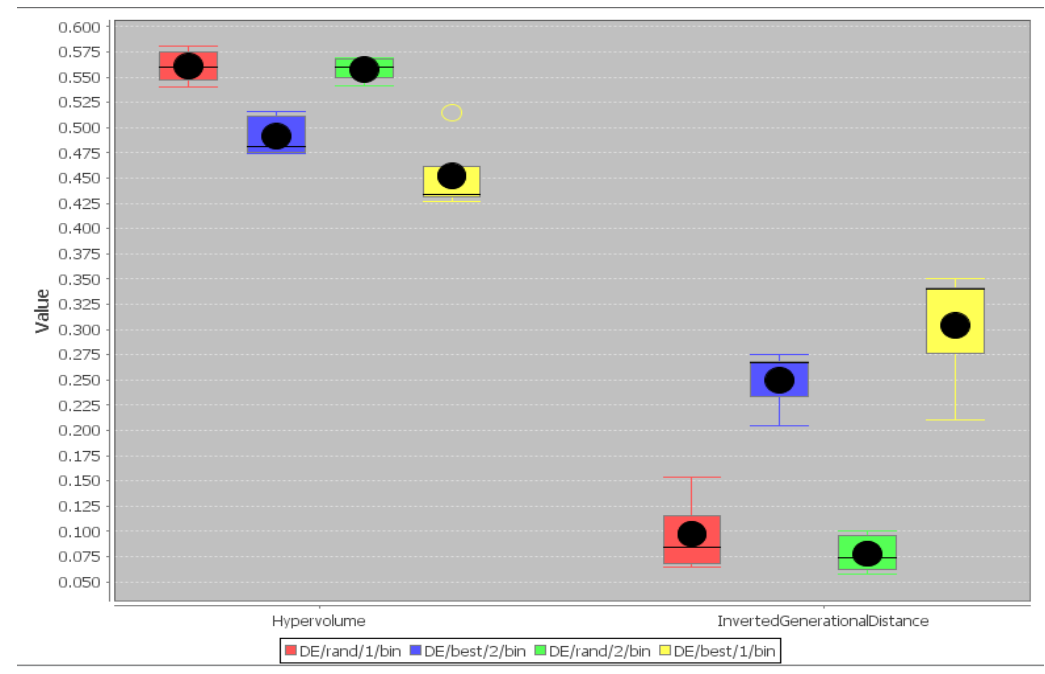

Figure 6. Comparison of DE variants with weighted sum

According to hyper volume the higher one performs better and according to IGD lower one performs better. Thus, based on the result obtained above DE/rand/2 performs better in weighted sum and according to IGD rand/2 performs better.

\subsubsection{Tchebysheff scalarization function comparison}

Figure 7 shows the results of different DE variants viz. DE/rand/1, DE/rand/2, DE/best/1 and DE/best/2, on HV and IGD metrics using the scalarizing function Tchebysheff.

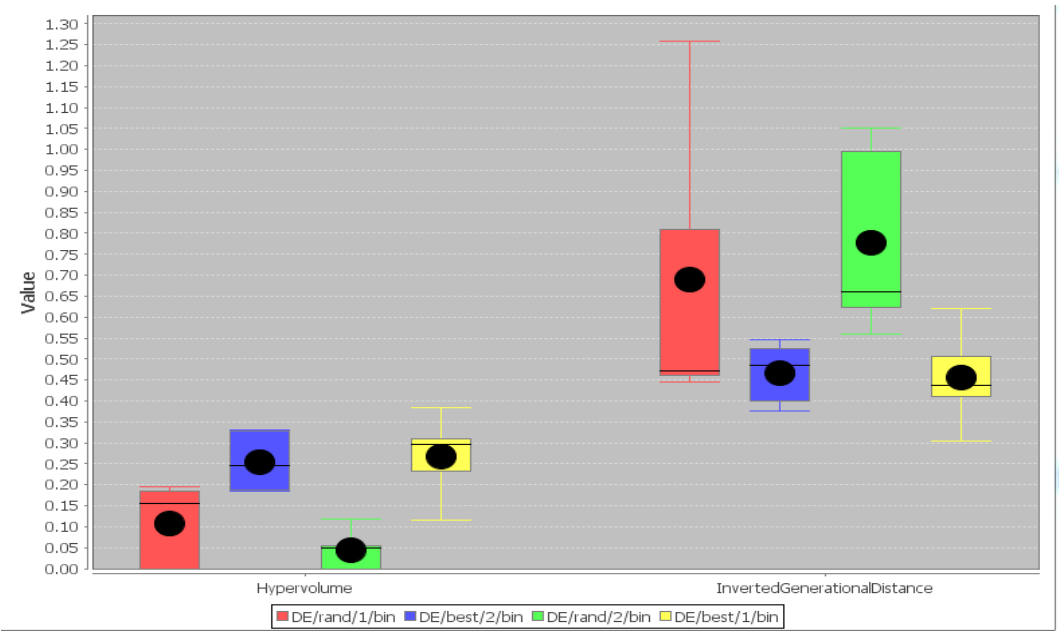

Figure 7. Comparison of DE variants with Tchebysheff 
According to IGD and hyper volume, DE/best/1 gets greater value on hyper volume and lower value on IGD thus producing better results.

\subsubsection{Modified Tchebysheff}

Figure 8 shows the results of different DE variants viz. DE/rand/1, DE/rand/2, DE/best/1 and DE/best/2, on HV and IGD metrics using the scalarizing function Modified Tchebysheff.

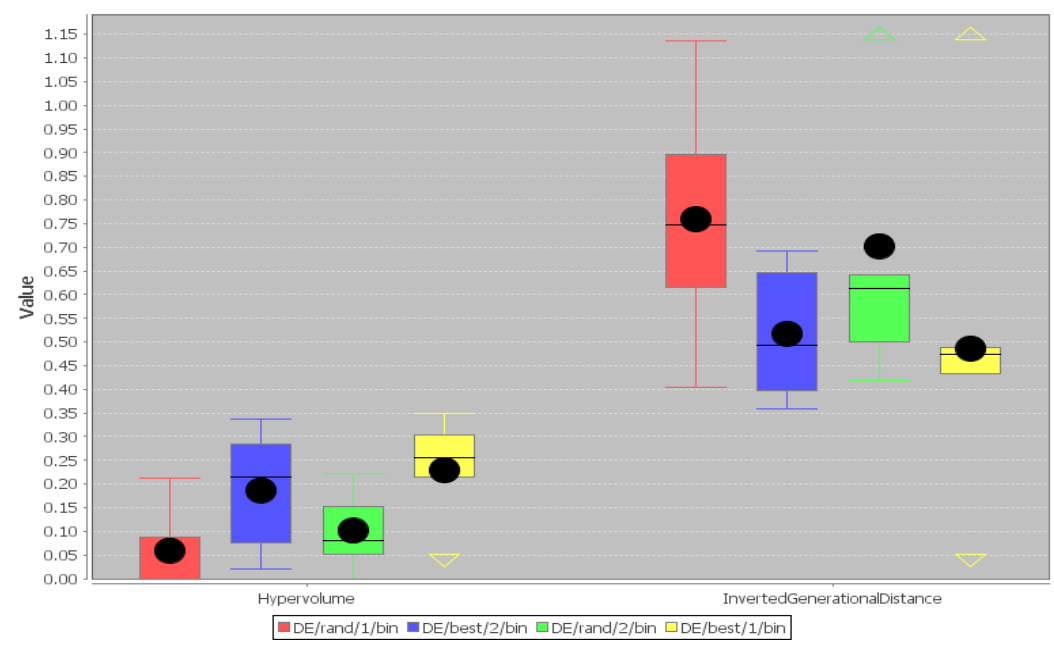

Figure 8. Comparison of DE variants with Modified Tchebysheff

From above result it is observed that on hyper volume DE/best/1 performs better and on IGD $\mathrm{DE} / \mathrm{best} / 2$ performs better thus one single DE variant cannot be said as better thus modified Tchebysheff cannot be said to perform better on a single DE variant.

\subsubsection{PBI}

Following Figure 9 shows the results of different DE variants viz. DE/rand/1, DE/rand/2, DE/best/1 and DE/best/2, on HV and IGD metrics using the scalarizing function PBI.

According to the obtained result on PBI, by using IGD best/2 performs well and hyper volume produces value in negative thus anything cannot be predicted. Thus corresponding to the found experimental results, Tchebysheff produces a better result as both hyper volume and IGD shows same DE variant i.e. $\mathrm{DE} / \mathrm{best} / 1$ and plot on function evaluation for Tchebysheff produces better results on DE/best/1. We conclude that Tchebysheff scalarization function performs better on MOP.

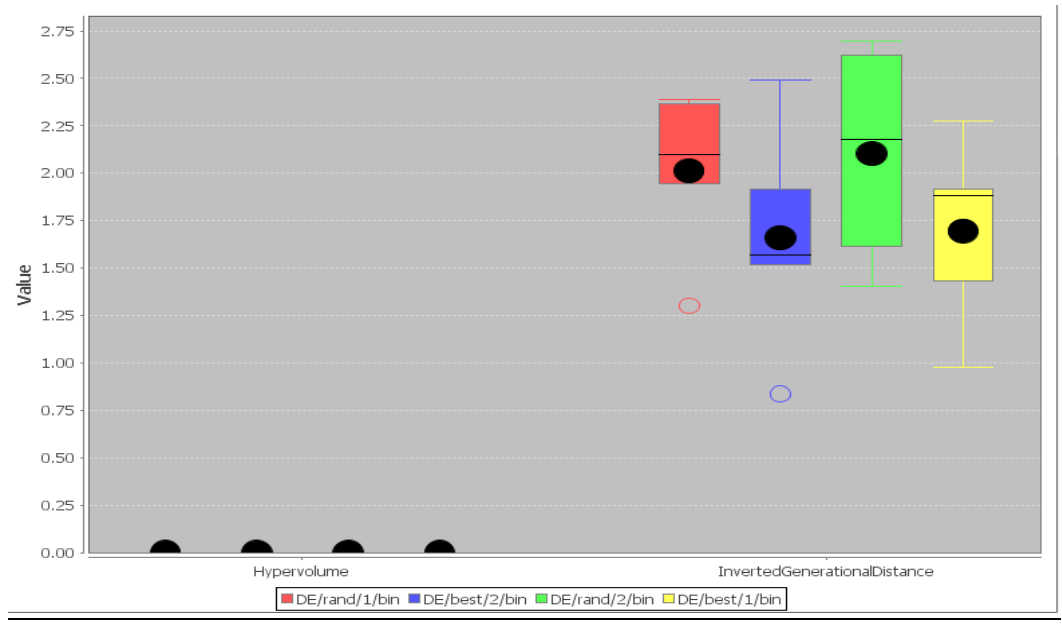

Figure 9. Comparison of DE variants with PBI 


\section{CONCLUSION}

The way the technology has shown growth in the past decade is tremendous and unbelievable[12]. The growth in Computing technologies, IoT, Artificial Intelligence, AR/VR etc. is amazing [13, 14]. If it is possible to map the given problem as optimization kind problem, then it is very well that differential evolution algorithm can be used to solve that. So alongside these, Evolutionary algorithms are also growing in parallel to solve multi-disciplinary problems.

This paper examines the performance of four different scalarizing functions by using four DE algorithm variants such as DE/rand/1, DE/rand/2, DE/best/1 and DE/best/2 for solving MOP. Experimental results shows the better performance of Tchebysheff scalarizing function as it produces consistent result on different indicators such as IGD and HV for the DE algorithm, DE/best/1. Moreover, other scalarizing functions does not produce consistent results on different indicators. In-depth analysis can be performed by including more MOPs, more indicator functions and more evolutionary algorithms in future work.

\section{REFERENCES}

[1] W. Huang H. Li. "On the differential evolution schemes in MOEA/D," Proc. 6th Int. Conf. Nat. Comput (ICNC) 2010; $6,2010$.

[2] David hadka. "MOEA Framework: A Free and Open Source Java Framework for Multiobjective Optimization". ; 2011-2016. http://moeaframework.org/downloads.html.

[3] Ma Et Al. "On Tchebycheff Decomposition Approaches for MOEA," IEEE transactions on evolutionary computation 2018; 22(2):

[4] S. Thangavelu \& C. Shunmuga Velayutham. "An investigation on mixing heterogeneous differential evolution variants in a distributed framework International". Journal of Bio-Inspired Computation. 2015; 7.

[5] Aswani V, Praveen V, Thangavelu S. "Performance analysis of variants of Differential Evolution on Multi-objective optimization problems". Indian Journal of Science and Technology 2015; 8(17):

[6] David hadka. Beginner's Guide to the MOEA Framework.. 2016. https://books.google.co.in/books?id=okUfjwEACAAJ.

[7] S. Jiang, S. Yang, Y. Wang, X. Liu. "Scalarizing functions in decomposition-based multiobjective evolutionary algorithms".IEEE transactions on evolutionary computation. 2017.

[8] Lotfi, S. \& Karimi, F. “A hybrid MOEA/DTS for Solving Multi-Objective Problems". Journal of AI \& Data Mining. Proc. 6th Int. Conf. Nat. Comput (ICNC) 2017; 5(2).

[9] David hadka. MOEA Framework: A Free and Open Source Java Framework for Multiobjective Optimization. 20112016

[10] K. V. Price. An introduction to differential evolution. In D.W. Corne, M. Dorigo, F. Glover (eds), "New Ideas in Optimization”. McGraw Hill, 1999.

[11] (2018) Test functions for optimization, Available at: https://en.wikipedia.org/wiki/Test_functions_for_optimization (Accessed: 19 April 2018)

[12] Vasudevan, S.K., Vivek, C. and Srivathsan, S. "An intelligent boxing application through augmented reality for two users-human computer interaction attempt". Indian Journal of Science and Technology., 8(34), 2015

[13] Abhishek, S.N., Vasudevan, S.K., Nair, P.R., Thangavelu, S. and Sundaram, R.M.D. "A Proposal for Mitigating Fishermen Killing In Indian Sea Borders through Technology-Maritime Boundary Identification Device". Indonesian Journal of Electrical Engineering and Computer Science 6(3), pp.704-710, 2017.

[14] Datta, B.S., Ganapathy, R., Vasudevan, S.K. and Abhishek, "An Inventive and Innovative Alternate for Legacy Chain Pulling System through Internet of Things". S.N. Indonesian Journal of Electrical Engineering and Computer Science, 6(3), pp.688-694, 2017. 